\title{
The Transmuted Generalized Inverse Weibull Distribution
}

\author{
Faton Merovci \\ University of Prishtina
}

\author{
Ibrahim Elbatal \\ Cairo University
}

\author{
Alaa Ahmed \\ Cairo University
}

\begin{abstract}
A generalization of the generalized inverse Weibull distribution the so-called transmuted generalized inverse Weibull distribution is proposed and studied. We will use the quadratic rank transmutation map (QRTM) in order to generate a flexible family of probability distributions taking the generalized inverse Weibull distribution as the base value distribution by introducing a new parameter that would offer more distributional flexibility. Various structural properties including explicit expressions for the moments, quantiles, and moment generating function of the new distribution are derived. We propose the method of maximum likelihood for estimating the model parameters and obtain the observed information matrix. A real data set are used to compare the flexibility of the transmuted version versus the generalized inverse Weibull distribution.
\end{abstract}

Keywords: generalized inverse Weibull distribution, order statistics, transmutation map, maximum likelihood estimation, reliability function.

\section{Introduction}

The inverse Weibull distribution is another life time probability distribution which can be used in the reliability engineering discipline. The inverse Weibull distribution can be used to model a variety of failure characteristics such as infant mortality, useful life and wear- out periods. It can also be used to determine the cost effectiveness, maintenance periods of reliability centered maintenance activities and applications in medicine, reliability and ecology. Keller, Goblin, and Farnworth (1985) obtained the inverse Weibull model by investigating failures of mechanical components subject to degradation. Drapella (1993); Mudholkar and Kollia (1994), and de Gusmão, Ortega, and Cordeiro (2011) introduced the generalized inverse Weibull distribution, among others. The cumulative distribution function (cdf) of the generalized inverse Weibull (GIW) distribution can be defined by

$$
G(x, \alpha, \gamma, \theta)=e^{-\gamma(\alpha x)^{-\beta}}, \quad \alpha>0, \gamma>0, \beta>0, x \geq 0,
$$

where $\alpha$ is a scale parameter and $\beta, \gamma$ are shape parameters, respectively. The corresponding probability density function (pdf) is given by

$$
g(x, \alpha, \gamma, \theta)=\alpha \beta \gamma(\alpha x)^{-\beta-1} e^{-\gamma(\alpha x)^{-\beta}} .
$$

In this article we present a new generalization of the generalized inverse Weibull distribution called the transmuted generalized inverse Weibull distribution. We will derive the subject distribution using the quadratic rank transmutation map studied by Shaw and Buckley (2009). 
A random variable $X$ is said to have transmuted distribution if its cdf is given by

$$
F(x)=(1+\lambda) G(x)-\lambda G(x)^{2}, \quad|\lambda| \leq 1,
$$

where $G(x)$ is the cdf of the base distribution, which on differentiation yields

$$
f(x)=g(x)[1+\lambda-2 \lambda G(x)],
$$

where $f(x)$ and $g(x)$ are the corresponding pdf's associated with the cdf's $F(x)$ and $G(x)$, respectively. An extensive information about the quadratic rank transmutation map is given in Shaw and Buckley (2009). Observe that at $\lambda=0$ we have the distribution of the base random variable.

Many authors deal with the generalization of some well-known distributions. Aryal and Tsokos (2009) defined the transmuted generalized extreme value distribution and they studied some basic mathematical characteristics of the transmuted Gumbel probability distribution and it has been observed that the transmuted Gumbel can be used to model climate data. Also Aryal and Tsokos (2011) presented a new generalization of the Weibull distribution called the transmuted Weibull distribution. Recently, Aryal (2013) proposed and studied various structural properties of the transmuted log-logistic distribution. Khan and King (2013) introduced the transmuted modified Weibull distribution which extended recent developments on the transmuted Weibull distribution by Aryal and Tsokos (2009). They studied the mathematical properties and the maximum likelihood estimation of the unknown parameters. In the present study we will provide the mathematical formulation of the transmuted generalized inverse Weibull distribution and some of its properties. We will also provide possible areas of applications.

The rest of the paper is organized as follows. In Section 3 we demonstrate the transmuted probability distribution. In Section 4 we find the reliability functions of the subject model. The statistical properties including quantile functions, moments and moment generating functions are derived in Section 5. The minimum, maximum, and median order statistics models are discussed in Section 6. Least squares and weighted least squares estimators are discussed in Section 7. In Section 8 we demonstrate the maximum likelihood estimates and some asymptotic confidence intervals for the unknown parameters. In Section 9, the TGIW distribution is applied to a real data set. Finally, we provide some conclusion in Section 10.

\section{Transmutation map}

In this section we consider the transmuted probability distribution. Let $F_{1}$ and $F_{2}$ be the cdf's of two distributions with a common sample space. The general rank transmutation as given in Shaw and Buckley (2009) is defined as

$$
G_{R 12}(u)=F_{2}\left(F_{1}^{-1}(u)\right), \quad G_{R 21}(u)=F_{1}\left(F_{2}^{-1}(u)\right) .
$$

Note that the inverse cdf is also known as the quantile function an is defined as

$$
F^{-1}(y)=\inf _{x \in \mathbb{R}}\{F(x) \geq y\} \quad \text { for } y \in[0,1] .
$$

The functions $G_{R 12}(u)$ and $G_{R 21}(u)$ both map the unit interval $I=[0,1]$ onto itself, and under suitable assumptions they are mutual inverses and satisfy $G_{R i j}(0)=0$ and $G_{R i j}(1)=1$. A quadratic rank transmutation map (QRTM) is defined as

$$
G_{R 12}(u)=u+\lambda u(1-u), \quad|\lambda| \leq 1,
$$

from which follows that the cdf's satisfy the relationship

$$
F_{2}(x)=(1+\lambda) F_{1}(x)-\lambda F_{1}(x)^{2},
$$

which after differentiation yields

$$
f_{2}(x)=f_{1}(x)\left[1+\lambda-2 \lambda F_{1}(x)\right],
$$


where $f_{1}(x)$ and $f_{2}(x)$ are the corresponding pdf's associated with the cdf's $F_{1}(x)$ and $F_{2}(x)$, respectively. An extensive information about the quadratic rank transmutation map is given in Shaw and Buckley (2009). Observe that at $\lambda=0$ we have the distribution of the base random variable. The function $f_{2}(x)$ in (7) satisfies the property of a pdf.

\section{Transmuted generalized inverse Weibull distribution}

In this section we study the transmuted generalized inverse Weibull (TGIW) distribution and submodels of this distribution. Now using (1) and (2) we have the cdf of the transmuted generalized inverse Weibull distribution

$$
F_{T G I W}(x)=e^{-\gamma(\alpha x)^{-\beta}}\left[1+\lambda-\lambda e^{-\gamma(\alpha x)^{-\beta}}\right],
$$

where $\alpha$ is a scale parameter and $\beta$ and $\gamma$ are shape parameters representing the different patterns of the transmuted generalized inverse Weibull distribution, and $\lambda$ is the transmuted parameter. The corresponding pdf of the transmuted generalized inverse Weibull distribution is given by

$$
f_{T G I W}(x)=\alpha \beta \gamma(\alpha x)^{-\beta-1} e^{-\gamma(\alpha x)^{-\beta}}\left[1+\lambda-2 \lambda e^{-\gamma(\alpha x)^{-\beta}}\right] .
$$

Figures 1 and 2 illustrate some of the possible shapes of the pdf and cdf of a TGIW distribution for selected values of the parameters $\beta, \gamma$, and $\lambda$ by keeping $\alpha=1$, respectively.

The transmuted generalized inverse Weibull distribution is a very flexible model that approaches to different distributions when its parameters are changed. The flexibility of the transmuted generalized inverse Weibull distribution is explained in the following. If $X$ is a random variable with pdf (9), then we have the following cases:

(a) If $\gamma=1$, we get the transmuted inverse Weibull.

(b) If $\lambda=0$ and $\gamma=1$, we get the inverse Weibull.

(c) If $\beta=1$ and $\gamma=1$, we get the transmuted inverse exponential distribution.

(d) If $\beta=1, \gamma=1$, and $\lambda=0$, we get the inverse exponential distribution.

(e) If $\beta=2$ and $\gamma=1$ we get transmuted inverse Rayleigh distribution.

(f) If $\beta=2, \gamma=1$, and $\lambda=0$ we get the inverse Rayleigh distribution.

(g) If $\alpha=1$ we get the transmuted Frechet distribution.

(h) If $\alpha=1$ and $\lambda=0$ we get the Frechet distribution.

\section{Reliability analysis}

The reliability function $R(x)$, which is the probability of an item not failing prior to some time $t$, is defined by $R(x)=1-F(x)$. The reliability function of a transmuted generalized inverse Weibull distribution $R_{T G I W}(x)$ can be a useful characterization of life time data analysis. It is defined as

$$
\begin{aligned}
R_{T G I W}(x) & =1-F_{T G I W}(x) \\
& =1-e^{-\gamma(\alpha x)^{-\beta}}\left[1+\lambda-\lambda e^{-\gamma(\alpha x)^{-\beta}}\right] .
\end{aligned}
$$

It is important to note that $R_{T G I W}(x)+F_{T G I W}(x)=1$. The other characteristic of interest is the hazard rate function defined by $h_{T G I W}(x)=f_{T G I W}(x) /\left(1-F_{T G I W}(x)\right)$, which is an important quantity characterizing life phenomenon. It can be loosely interpreted as the conditional probability 


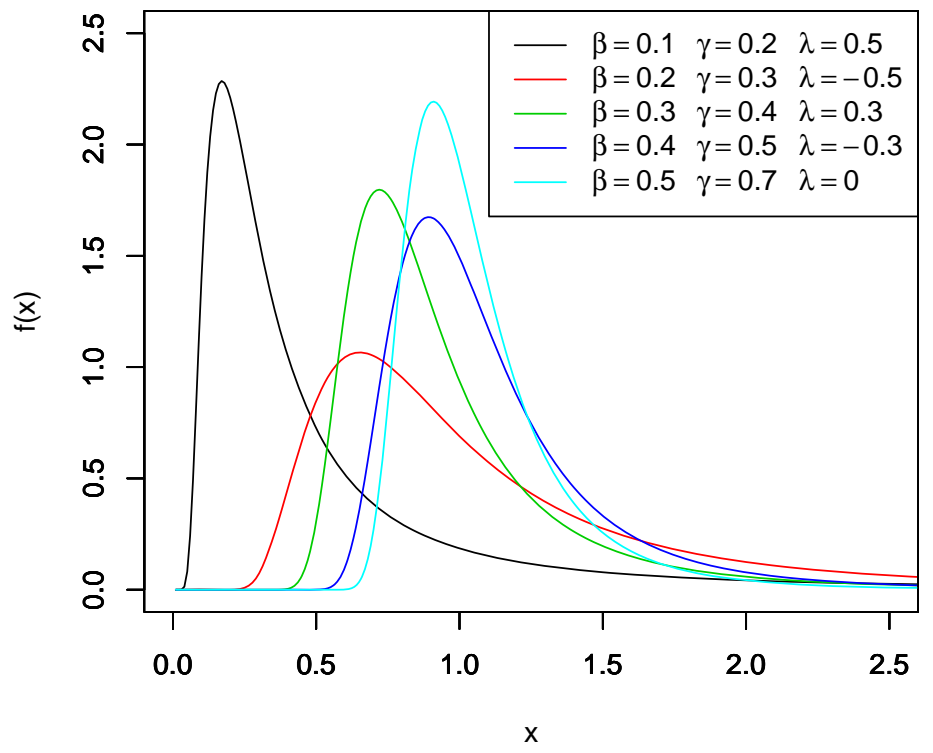

Figure 1: The pdf's of various TGIW distributions.

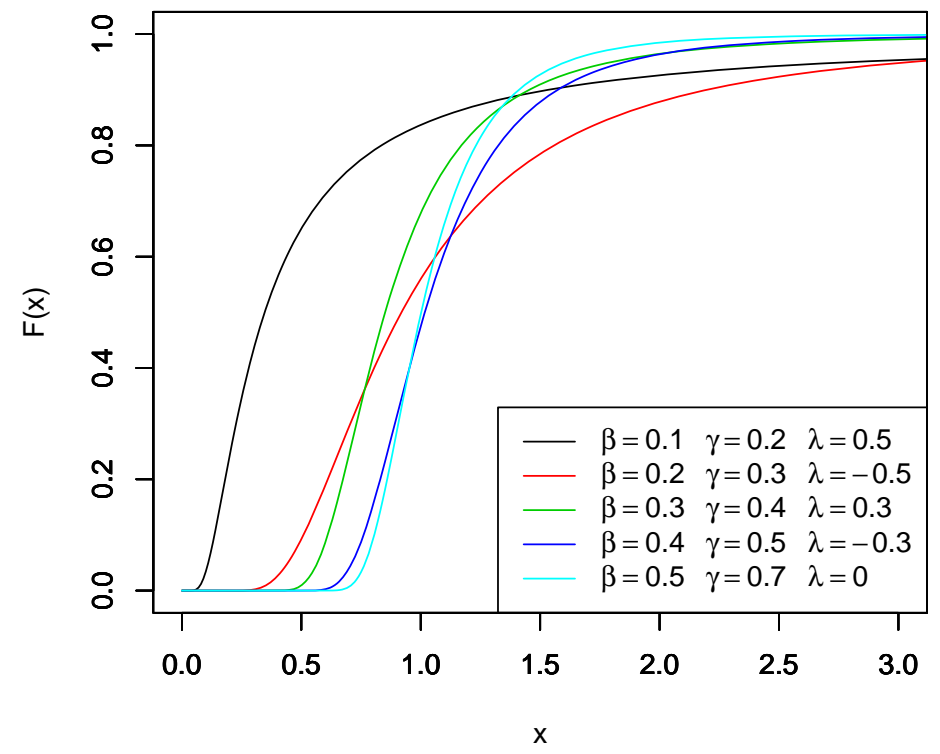

Figure 2: The cdf's of various TGIW distributions.

of failure, given it has survived to the time $t$. The hazard rate function for a transmuted generalized 
inverse Weibull distribution is defined by

$$
\begin{aligned}
h_{T G I W}(x) & =\frac{f_{T G I W}(x)}{1-F_{T G I W}(x)} \\
& =\frac{\alpha \beta \gamma(\alpha x)^{-\beta-1} e^{-\gamma(\alpha x)^{-\beta}}\left[1+\lambda-2 \lambda e^{-\gamma(\alpha x)^{-\beta}}\right]}{1-e^{-\gamma(\alpha x)^{-\beta}}\left[1+\lambda-\lambda e^{-\gamma(\alpha x)^{-\beta}}\right]} .
\end{aligned}
$$

Figures 3 and 4 illustrate some of the possible shapes of the hazard rate function and the survival function of the TGIW distribution for selected values of the parameters $\beta, \gamma$, and $\lambda$ by keeping $\alpha=1$, respectively.

It is important to note that the unit for $h_{T G I W}(x)$ is the probability of failure per unit of time, distance or cycles. These failure rates are defined with different choices of parameters. The cumulative hazard function of the transmuted generalized inverse Weibull distribution is denoted by

$$
H_{T G I W}(x)=-\log \left|e^{-\gamma(\alpha x)^{-\beta}}\left[1+\lambda-\lambda e^{-\gamma(\alpha x)^{-\beta}}\right]\right|
$$

It is important to note that the unit for $H_{T G I W}(x)$ is the cumulative probability of failure per unit of time, distance or cycles.

\section{Statistical properties}

In this section we discuss the statistical properties of the transmuted generalized inverse Weibull distribution. Specifically we are interested in quantiles, a random number generation function, moments and the moment generating function.

\subsection{Quantiles}

The quantile $x_{q}$ of the $T_{G I W}(\alpha, \beta, \gamma, \lambda, x)$ distribution is the solution of the equation

$$
x_{q}=\frac{1}{\alpha}\left[\frac{1}{\gamma} \log \left(\frac{1+\lambda-\lambda e^{-\gamma\left(\alpha x_{q}\right)^{-\beta}}}{q}\right)\right]^{-\frac{1}{\beta}} .
$$

The above equation has no closed form solution in $x_{q}$, so we have to use a numerical technique such as a Newton-Raphson method to get the quantile. If we put $q=0.5$ in equation (13) one gets the median.

\subsection{Random number generation}

A random variate $X$ from $T_{G I W}(\alpha, \beta, \gamma, \lambda, x)$ can be generated as $x_{U}$ according to (13), where $q$ is replaced by $U \sim U(0,1)$.

\subsection{Moments}

The following theorem gives the $r$ th moment $\mu_{r}^{\prime}$ of the $T_{G I W}(\alpha, \beta, \gamma, \lambda, x)$ distribution.

Theorem 4.1. If $X$ is from the $T_{G I W}(\alpha, \beta, \gamma, \lambda, x)$ distribution with $|\lambda| \leq 1$, then the $r$ th non central moments are given by

$$
\mu_{r}^{\prime}=\mathrm{E}\left(X^{r}\right)=\frac{\gamma^{r / \beta} \Gamma(1-r / \beta)}{\alpha^{r}}\left[1+\lambda-\lambda 2^{r / \beta}\right]
$$




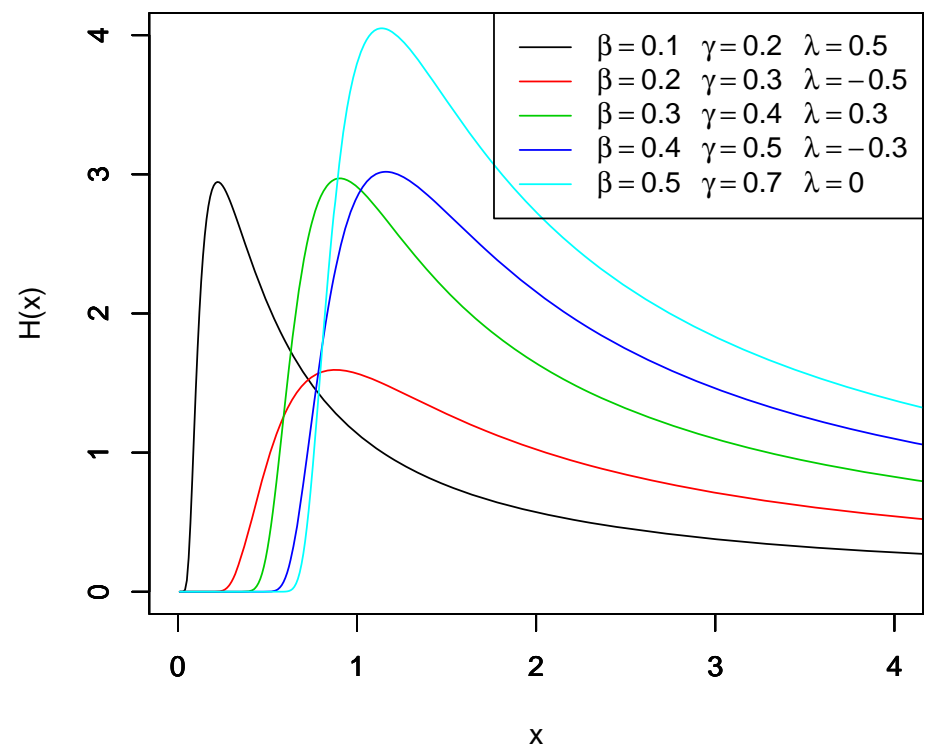

Figure 3: The hazard function of various TGIW distributions.

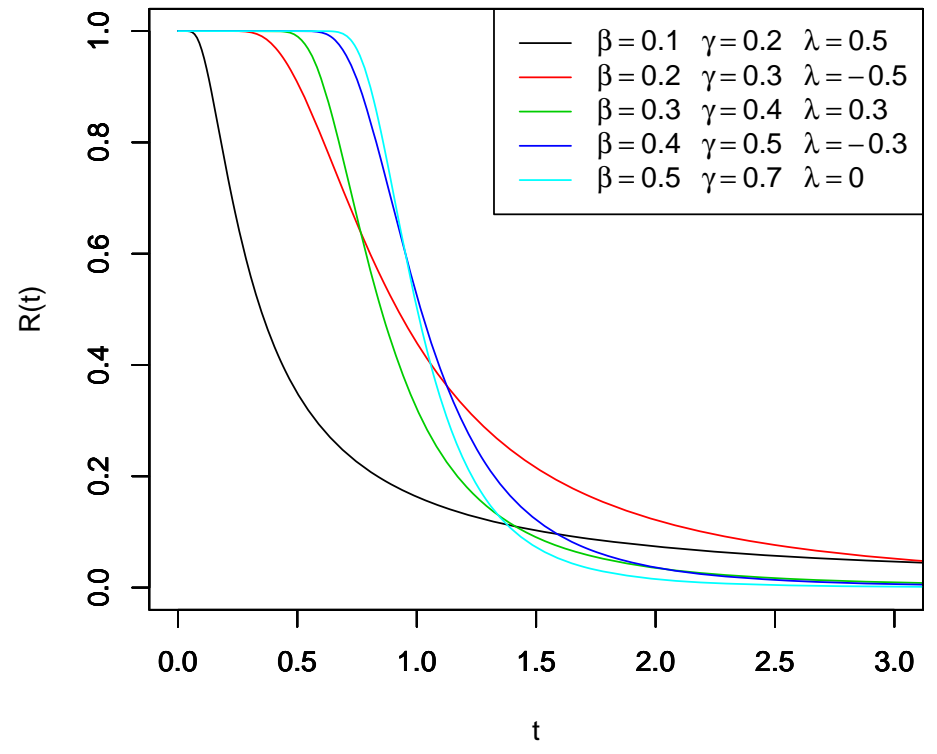

Figure 4: The survival function of various TGIW distributions.

Proof: Starting with

$$
\begin{aligned}
\mu_{r}^{\prime} & =\int_{0}^{\infty} x^{r} f_{T G I W}(\alpha, \beta, \gamma, \lambda, x) d x \\
& =\int_{0}^{\infty} x^{r} \alpha \beta \gamma(\alpha x)^{-\beta-1} e^{-\gamma(\alpha x)^{-\beta}}\left[1+\lambda-2 \lambda e^{-\gamma(\alpha x)^{-\beta}}\right] d x \\
& =\frac{\alpha \beta \gamma}{\alpha^{r}}(1+\lambda) \int_{0}^{\infty}(\alpha x)^{r-\beta-1} e^{-\gamma(\alpha x)^{-\beta}} d x-\frac{2 \lambda \alpha \beta \gamma}{\alpha^{r}} \int_{0}^{\infty}(\alpha x)^{r-\beta-1} e^{-2 \gamma(\alpha x)^{-\beta}} d x .(15)
\end{aligned}
$$


Now let $\gamma(\alpha x)^{-\beta}=t$, then $x=\frac{1}{\alpha} \gamma^{1 / \beta} t^{-1 / \beta}$, therefore

$$
\begin{aligned}
\mu_{r}^{\prime} & =\frac{1+\lambda}{\alpha^{r}} \gamma^{r / \beta} \Gamma(1-r / \beta)-\frac{\lambda}{\alpha^{r}}(2 \gamma)^{r / \beta} \Gamma(1-r / \beta) \\
& =\frac{\gamma^{r / \beta} \Gamma(1-r / \beta)}{\alpha^{r}}\left[1+\lambda-\lambda 2^{r / \beta}\right]
\end{aligned}
$$

which completes the proof.

Based on Theorem 4.1 the coefficients of variation (CV), skewness (CS), and kurtosis (CK) can be obtained according to the following well-known relations as

$$
\begin{aligned}
\mathrm{CV}_{\text {TMIW }} & =\sqrt{\frac{\mu_{2}}{\mu_{1}}-1} \\
\mathrm{CS}_{\text {TMIW }} & =\frac{\mu_{3}-3 \mu_{2} \mu_{1}+2 \mu_{1}^{3}}{\left(\mu_{2}-\mu_{1}\right)^{3 / 2}} \\
\mathrm{CK}_{T M I W} & =\frac{\mu_{4}-4 \mu_{3} \mu_{1}+6 \mu_{2} \mu_{1}^{2}}{\left(\mu_{2}-\mu_{1}^{2}\right)^{2}} .
\end{aligned}
$$

\subsection{Moment generating function}

In this subsection we derive the moment generating function (mgf) of the transmuted generalized inverse Weibull distribution.

Theorem 4.2. If $X$ has the $T_{G I W}(\alpha, \beta, \gamma, \lambda, x)$ distribution with $|\lambda| \leq 1$, then the moment generating function (mgf) of $X$ is given as

$$
M_{X}(t)=\sum_{r=0}^{\infty} \frac{t^{r} \gamma^{r / \beta} \Gamma(1-r / \beta)}{r ! \alpha^{r}}\left[1+\lambda-\lambda 2^{r / \beta}\right] .
$$

Proof:

$$
\begin{aligned}
M_{X}(t) & =\int_{0}^{\infty} e^{t x} f_{T_{G I W}}(\alpha, \beta, \gamma, \lambda, x) d x \\
& =\int_{0}^{\infty} \sum_{r=0}^{\infty} \frac{t^{r}}{r !} x^{r} f_{T_{G I W}}(\alpha, \beta, \gamma, \lambda, x) \\
& =\sum_{r=0}^{\infty} \frac{t^{r}}{r !} \mu_{r}^{\prime} .
\end{aligned}
$$

By using equation (14) in result (18) we get

$$
M_{X}(t)=\sum_{r=0}^{\infty} \frac{t^{r} \gamma^{r / \beta} \Gamma(1-r / \beta)}{r ! \alpha^{r}}\left[1+\lambda-\lambda 2^{r / \beta}\right],
$$

which completes the proof.

\section{Order statistics}

In fact, the order statistics have many applications in reliability and life testing. The order statistics arise in the study of reliability of a system. Let $X_{1}, \ldots, X_{n}$ be a simple random sample from the $T_{G I W}(\alpha, \beta, \gamma, \lambda, x)$ distribution with cdf and pdf as in (8) and (9), respectively. Let $X_{(1: n)} \leq \cdots \leq$ $X_{(n: n)}$ denote the order statistics obtained from this sample. In reliability literature, $X_{(i: n)}$ denotes 
the lifetime of an $(n-i+1)$-out-of- $n$ system which consists of $n$ iid components. Then, the pdf of $X_{(i: n)}, 1=1, \ldots, n$ is

$$
f_{i: n}(x)=\frac{1}{\operatorname{Beta}(i, n-i+1)}[F(x, \Phi)]^{i-1}[1-F(x, \Phi)]^{n-i} f(x, \Phi),
$$

where $\Phi=(\alpha, \beta, \gamma, \lambda)$. Also, the joint pdf of $\left(X_{(i: n)}, X_{(j: n)}\right)$, for $i=1, \ldots, n$, is

$$
f_{i: j: n}\left(x_{i}, x_{j}\right)=C\left[F\left(x_{i}\right)\right]^{i-1}\left[F\left(x_{j}\right)-F\left(x_{i}\right)\right]^{j-i-1}\left[1-F\left(x_{j}\right)\right]^{n-j} f\left(x_{i}\right) f\left(x_{j}\right),
$$

where

$$
C=\frac{n !}{(i-1) !(j-i-1) !(n-j) !} .
$$

We define the first order statistics as $X_{(1)}=\min \left(X_{1}, \ldots, X_{n}\right)$, the last order statistics as $X_{(n)}=$ $\max \left(X_{1}, \ldots, X_{n}\right)$, and the median order as $X_{m+1}$, if $n=2 m+1$.

\subsection{Distribution of minimum, maximum, and median}

Let $X_{1}, \ldots, X_{n}$ be independently identically distributed random variables from the transmuted generalized inverse Weibull distribution. The first, last, and median order pdf's are given by

$$
\begin{aligned}
f_{1: n}(x)= & n[1-F(x, \Phi)]^{n-1} f(x, \Phi) \\
= & n\left\{1-e^{-\gamma(\alpha x)^{-\beta}}\left[1+\lambda-\lambda e^{-\gamma(\alpha x)^{-\beta}}\right]\right\}^{n-1} \\
& \times \alpha \beta \gamma(\alpha x)^{-\beta-1} e^{-\gamma(\alpha x)^{-\beta}}\left[1+\lambda-2 \lambda e^{-\gamma(\alpha x)^{-\beta}}\right] \\
f_{n: n}(x)= & \left.n[F(x, \Phi)]^{n-1} f(x), \Phi\right) \\
= & n\left\{e^{-\gamma(\alpha x)^{-\beta}}\left[1+\lambda-\lambda e^{-\gamma(\alpha x)^{-\beta}}\right]\right\}^{n-1} \\
& \times \alpha \beta \gamma(\alpha x)^{-\beta-1} e^{-\gamma(\alpha x)^{-\beta}}\left[1+\lambda-2 \lambda e^{-\gamma(\alpha x)^{-\beta}}\right] \\
f_{m+1: n}(x)= & \frac{(2 m+1) !}{m ! m !}(F(x))^{m}(1-F(x))^{m} f(x) \\
& \frac{(2 m+1) !}{m ! m !}\left\{e^{-\gamma(\alpha x)^{-\beta}}\left[1+\lambda-\lambda e^{-\gamma(\alpha x)^{-\beta}}\right]\right\}^{m} \\
& \times\left\{1-e^{-\gamma(\alpha x)^{-\beta}}\left[1+\lambda-\lambda e^{-\gamma(\alpha x)^{-\beta}}\right]\right\}^{m} \\
& \times \alpha \beta \gamma(\alpha x)^{-\beta-1} e^{-\gamma(\alpha x)^{-\beta}}\left[1+\lambda-2 \lambda e^{-\gamma(\alpha x)^{-\beta}}\right] .
\end{aligned}
$$

\subsection{Joint distribution of the $i$ th and $j$ th order statistics}

The joint distribution of the the $i$ th and $j$ th order statistics from the transmuted generalized inverse Weibull is

$$
\begin{aligned}
f_{i: j: n}\left(x_{i}, x_{j}\right)= & C\left[F\left(x_{i}\right)\right]^{i-1}\left[F\left(x_{j}\right)-F\left(x_{i}\right)\right]^{j-i-1}\left[1-F\left(x_{j}\right)\right]^{n-j} f\left(x_{i}\right) f\left(x_{j}\right) \\
= & C\left\{h_{i}\left[1+\lambda-\lambda h_{i}\right]\right\}^{i-1} \\
& \times\left\{h_{j}\left[1+\lambda-\lambda h_{j}\right]-h_{i}\left[1+\lambda-\lambda h_{i}\right]\right\}^{j-i-1} \\
& \times\left\{1-h_{j}\left[1+\lambda-\lambda h_{j}\right]\right\}^{n-j} \\
& \times \alpha \beta \gamma\left(\alpha x_{i}\right)^{-\beta-1} h_{i}\left[1+\lambda-2 \lambda h_{i}\right] \\
& \times \alpha \beta \gamma\left(\alpha x_{j}\right)^{-\beta-1} h_{j}\left[1+\lambda-2 \lambda h_{j}\right]
\end{aligned}
$$

where

$$
h_{i}=e^{-\gamma\left(\alpha x_{i}\right)^{-\beta}} .
$$


For the special case $i=1$ and $j=n$ we get the joint distribution of the minimum and maximum as

$$
\begin{aligned}
f_{1: n: n}\left(x_{1}, x_{n}\right)= & n(n-1)\left[F\left(x_{n}\right)-F\left(x_{1}\right)\right]^{n-2} f\left(x_{1}\right) f\left(x_{n}\right) \\
= & n(n-1)\left\{h_{n}\left[1+\lambda-\lambda h_{n}\right]-h_{1}\left[1+\lambda-\lambda h_{1}\right]\right\}^{n-2} \\
& \times \alpha \beta \gamma\left(\alpha x_{1}\right)^{-\beta-1} h_{1}\left[1+\lambda-2 \lambda h_{1}\right] \\
& \times \alpha \beta \gamma\left(\alpha x_{n}\right)^{-\beta-1} h_{n}\left[1+\lambda-2 \lambda h_{n}\right] .
\end{aligned}
$$

\section{Weighted least squares estimators}

In this section we provide the regression based method estimators of the unknown parameters of the transmuted generalized inverse Weibull distribution, which was originally suggested by Swain, Venkatraman, and Wilson (1988) to estimate the parameters of the beta distribution. It can be also used for some other cases. Suppose $X_{1}, \ldots, X_{n}$ is a random sample of size $n$ from a cdf $G(\cdot)$ and suppose $X_{(i)}, i=1, \ldots, n$, denotes the ordered sample. The proposed method uses the distribution of $G\left(X_{(i)}\right)$, which is $\operatorname{Beta}(i, n)$. Hence, for a sample of size $n$, we have

$$
\begin{aligned}
\mathrm{E}\left(G\left(X_{(j)}\right)\right) & =\frac{j}{n+1} \\
\operatorname{var}\left(G\left(X_{(j)}\right)\right) & =\frac{j(n-j+1)}{(n+1)^{2}(n+2)} \\
\operatorname{cov}\left(G\left(X_{(j)}\right), G\left(X_{(k)}\right)\right) & =\frac{j(n-k+1)}{(n+1)^{2}(n+2)}, \quad \text { for } j<k,
\end{aligned}
$$

see Johnson, Kotz and Balakrishnan Johnson, Kotz, and Balakrishnan (1994). Using the expectations and the variances, two variants of the least squares methods can be used.

Method 1 (Least Squares Estimators) Obtain the least squares estimators by minimizing

$$
\sum_{j=1}^{n}\left(G\left(X_{(j)}\right)-\frac{j}{n+1}\right)^{2}
$$

with respect to the unknown parameters. Therefore, in case of the $T G I W$ distribution the least squares estimators of $\alpha, \beta$, and $\lambda$, say $\hat{\alpha}_{L S}, \hat{\beta}_{L S}$, and $\hat{\lambda}_{L S}$, can be obtained by minimizing

$$
\sum_{j=1}^{n}\left[e^{-\gamma\left(\alpha x_{(j)}\right)^{-\beta}}\left[1+\lambda-\lambda e^{-\gamma\left(\alpha x_{(j)}\right)^{-\beta}}\right]-\frac{j}{n+1}\right]^{2},
$$

with respect to $\alpha, \beta$, and $\lambda$.

Method 2 (Weighted Least Squares Estimators) The weighted least squares estimators can be obtained by minimizing

$$
\sum_{j=1}^{n} w_{j}\left(G\left(X_{(j)}\right)-\frac{j}{n+1}\right)^{2},
$$

with respect to the unknown parameters, where

$$
w_{j}=\frac{1}{\operatorname{var}\left(G\left(X_{(j)}\right)\right)}=\frac{(n+1)^{2}(n+2)}{j(n-j+1)} .
$$

Therefore, in case of the TGIW distribution the weighted least squares estimators of $\alpha, \beta$, and $\lambda$, say $\hat{\alpha}_{W L S}, \hat{\beta}_{W L S}$, and $\hat{\lambda}_{W L S}$ can be obtained by minimizing

$$
\sum_{j=1}^{n} w_{j}\left[e^{-\gamma\left(\alpha x_{(j)}\right)^{-\beta}}\left[1+\lambda-\lambda e^{-\gamma\left(\alpha x_{(j)}\right)^{-\beta}}\right]-\frac{j}{n+1}\right]^{2}
$$


with respect to the unknown parameters.

\section{Maximum likelihood estimators}

Now we derive the maximum likelihood estimators (MLEs) and discuss inference under the $T_{G I W}(\alpha, \beta, \gamma, \lambda, x)$ distribution. Let $X_{1}, \ldots, X_{n}$ be a random sample of size $n$ from the $T_{G I W}$ distribution then the likelihood function can be written as

$$
L(\alpha, \beta, \gamma, \lambda, x)=\prod_{i=1}^{n} \alpha \beta \gamma\left(\alpha x_{i}\right)^{-\beta-1} e^{-\gamma\left(\alpha x_{i}\right)^{-\beta}}\left[1+\lambda-2 \lambda e^{-\gamma\left(\alpha x_{i}\right)^{-\beta}}\right] .
$$

Taking the logarithm results in the log-likelihood function

$$
\begin{aligned}
\log L= & n \log (\alpha \beta \gamma)-(\beta+1) \sum_{i=1}^{n} \log \left(\alpha x_{i}\right) \\
& -\gamma \sum_{i=1}^{n}\left(\alpha x_{i}\right)^{-\beta}+\sum_{i=1}^{n} \log \left[1+\lambda-2 \lambda e^{-\gamma\left(\alpha x_{i}\right)^{-\beta}}\right] .
\end{aligned}
$$

Differentiating equation (29) with respect to $\alpha, \beta, \gamma$, and $\lambda$ results in

$$
\begin{aligned}
\frac{\partial \log L}{\partial \alpha}= & \frac{n}{\alpha}-(\beta+1) \frac{n}{\alpha}+\gamma \beta \sum_{i=1}^{n} x_{i}\left(\alpha x_{i}\right)^{-\beta-1} \\
& -\sum_{i=1}^{n} \frac{2 \lambda \alpha x_{i}\left(\alpha x_{i}\right)^{-\beta-1} e^{-\gamma\left(\alpha x_{i}\right)^{-\beta}}}{1+\lambda-2 \lambda e^{-\gamma\left(\alpha x_{i}\right)^{-\beta}}}, \\
\frac{\partial \log L}{\partial \beta}= & \frac{n}{\beta}-\sum_{i=1}^{n} \log \left(\alpha x_{i}\right)+\gamma \sum_{i=1}^{n}\left(\alpha x_{i}\right)^{-\beta} \log \left(\alpha x_{i}\right) \\
& +\sum_{i=1}^{n} \frac{-2 \lambda \gamma e^{-\gamma\left(\alpha x_{i}\right)^{-\beta}}\left(\alpha x_{i}\right)^{-\beta} \log \left(\alpha x_{i}\right)}{1+\lambda-2 \lambda e^{-\gamma\left(\alpha x_{i}\right)^{-\beta}}}, \\
\frac{\partial \log L}{\partial \gamma}= & \frac{n}{\gamma}-\sum_{i=1}^{n}\left(\alpha x_{i}\right)^{-\beta}+\sum_{i=1}^{n} \frac{2 \lambda e^{-\gamma\left(\alpha x_{i}\right)^{-\beta}}\left(\alpha x_{i}\right)^{-\beta}}{1+\lambda-2 \lambda e^{-\gamma\left(\alpha x_{i}\right)^{-\beta}}}, \\
\frac{\partial \log L}{\partial \lambda}= & \sum_{i=1}^{n} \frac{1-2 e^{-\gamma\left(\alpha x_{i}\right)^{-\beta}}}{1+\lambda-2 \lambda e^{-\gamma\left(\alpha x_{i}\right)^{-\beta}} .}
\end{aligned}
$$

We can find estimates of the unknown parameters by the maximum likelihood method equating all the above nonlinear terms to zero and solving these equations simultaneously. The solutions are the MLE's $\hat{\alpha}, \hat{\beta}, \hat{\gamma}$, and $\hat{\lambda}$. For the three parameter transmuted generalized inverted Weibull distribution all second order derivatives exist. Thus we have

$$
(\hat{\alpha}, \hat{\beta}, \hat{\gamma}, \hat{\lambda})^{T} \sim \operatorname{Normal}\left((\alpha, \beta, \gamma, \lambda)^{T}, V^{-1}\right)
$$

with the symmetric matrix

$$
V=-E\left[\begin{array}{cccc}
V_{\alpha \alpha} & V_{\alpha \beta} & V_{\alpha \gamma} & V_{\alpha \lambda} \\
& V_{\beta \beta} & V_{\beta \gamma} & V_{\beta \lambda} \\
& & V_{\gamma \gamma} & V_{\gamma \lambda} \\
& & & V_{\lambda \lambda}
\end{array}\right],
$$

where $V_{\theta_{1} \theta_{2}}=\partial^{2} L / \partial \theta_{1} \partial \theta_{2}$ denotes the second derivative of $L$ with respect to $\theta_{1}$ and $\theta_{2}$. The matrix $V^{-1}$ represents the asymptotic variance/covariance matrix of the MLE's. Based on (34), approximate $100(1-\delta) \%$ confidence intervals are determined as

$$
\hat{\alpha} \pm z_{1-\delta / 2} \sqrt{\hat{V}_{\alpha \alpha}}, \quad \hat{\beta} \pm z_{1-\delta / 2} \sqrt{\hat{V}_{\beta \beta}}, \quad \hat{\gamma} \pm z_{1-\delta / 2} \sqrt{\hat{V}_{\gamma \gamma}}, \quad \hat{\lambda} \pm z_{1-\delta / 2} \sqrt{\hat{V}_{\lambda \lambda}},
$$


where $z_{1-\delta}$ is the $100(1-\delta) \%$ percentile of the standard normal distribution and $\hat{V}$.. denotes the element of $V$.. evaluated in the MLE's.

We can compute the maximized unrestricted and restricted log-likelihood functions to construct the likelihood ratio test (LRT) statistic for testing some transmuted GIW sub-models. For example, we can use the LRT statistic to check whether the TGIW distribution for a given data set is statistically superior to the GIW distribution. In any case, hypothesis tests of the type $H_{0}: \theta=\theta_{0}$ versus $H_{1}: \theta \neq \theta_{0}$ can be performed using a LRT. In this case, the LRT statistic is $\omega=2(\log L(\hat{\theta}, x)-$ $\left.\log L\left(\hat{\theta}_{0}, x\right)\right)$, where $\hat{\theta}$ denotes the unrestricted MLE and $\hat{\theta}_{0}$ is the MLE under $H_{0}$. The LRT statistic $\omega$ is asymptotically (as $n \rightarrow \infty$ ) distributed as $\chi_{k}^{2}$, where $k$ is the number of parameters specified under $H_{0}$. The LRT rejects $H_{0}$ if $\omega>\chi_{k ; 1-\delta}^{2}$, where $\chi_{k ; 1-\delta}^{2}$ denotes the $100 \delta \%$ quantile of the $\chi_{k}^{2}$ distribution.

\section{Application}

Now we use real data to show that the TGIW distribution might fit better than a model based on the GIW distribution. The data set given in Table 1 is taken from Murthy, Xie, and Jiang (2004) page 180 and represents 50 items put into use at $t=0$ and failure times are in weeks.

Table 1: 50 items put into use at $t=0$ and their failure times in weeks.

\begin{tabular}{rrrrrrrrrr}
\hline 0.013 & 0.065 & 0.111 & 0.111 & 0.163 & 0.309 & 0.426 & 0.535 & 0.684 & 0.747 \\
0.997 & 1.284 & 1.304 & 1.647 & 1.829 & 2.336 & 2.838 & 3.269 & 3.977 & 3.981 \\
4.520 & 4.789 & 4.849 & 5.202 & 5.291 & 5.349 & 5.911 & 6.018 & 6.427 & 6.456 \\
6.572 & 7.023 & 7.087 & 7.291 & 7.787 & 8.596 & 9.388 & 10.261 & 10.713 & 11.658 \\
13.006 & 13.388 & 13.842 & 17.152 & 17.283 & 19.418 & 23.471 & 24.777 & 32.795 & 48.105 \\
\hline
\end{tabular}

Table 2: Parameter estimates of the GIW and TGIW distribution for 50 items put into use at $t=0$ and their failure times in weeks.

\begin{tabular}{llc}
\hline Model & Parameter Estimate & $-\log L(\cdot ; x)$ \\
\hline GIW & $\hat{\alpha}=0.854, \hat{\beta}=0.479$ & 168.638 \\
& $\hat{\gamma}=1.044$ & \\
\hline TGIW & $\hat{\alpha}=2.383, \hat{\beta}=0.530$ & 166.387 \\
& $\hat{\gamma}=1.143, \hat{\lambda}=-0.747$ & \\
\hline
\end{tabular}

Table 3: Goodness of fit criteria.

\begin{tabular}{lllll}
\hline Model & K-S & $-2 \log L$ & AIC & AICC \\
\hline GIW & 0.199 & 337.276 & 343.276 & 343.797 \\
TGIW & 0.192 & 332.774 & 340.774 & 341.662 \\
\hline
\end{tabular}

The LRT statistic to test $H_{0}: \lambda=0$ versus $H_{1}: \lambda \neq 0$ gives $\omega=4.502>3.841=\chi_{1 ; 0.95}^{2}$, so we reject the null hypothesis. In order to compare the two distribution models we consider criteria like the Kolmogorov-Smirnov test statistic, $-2 \log L$, the AIC (Akaike information criterion) as also the AICC (corrected AIC). The better distribution corresponds to smaller criterion values of AIC = $-2 \log L+2 k$ and AICC $=$ AIC $+\frac{2 k(k+1)}{n-k-1}$, where $k$ is the number of parameters in the statistical model, $n$ denotes the sample size and $\log L$ is the maximized value of the $\log$-likelihood function under the considered model. Table 2 shows the MLEs under both models, Table 3 contains the values the Kolmogorov-Smirnov test statistic, $-2 \log L$, AIC and AICC. These values indicate that the TGIW distribution leads to a better fit than the GIW model. 


\section{Conclusion}

Here we propose a new model, the so-called transmuted generalized inverse Weibull distribution which extends the generalized inverse Weibull distribution in the analysis of data with real support. An obvious reason for generalizing a standard distribution is because the generalized form provides more flexibility in modeling real data. We derive expansions for moments and for the moment generating function. The estimation of parameters is approached by the method of maximum likelihood, also the information matrix is derived. An application of the TGIW distribution to real data show that the new distribution can be used quite effectively to provide better fits than the GIW distribution.

\section{Acknowledgements}

The author would like to thank the Editor Herwig Friedl and the referee for carefully reading the manuscript and for their comments which greatly improved the presentation.

\section{References}

Aryal GR (2013). “Transmuted Log-Logistic Distribution.” Journal of Statistics Applications \& Probability, 2, 11-20.

Aryal GR, Tsokos CP (2009). "On the Transmuted Extreme Value Distribution with Application." Nonlinear Analysis: Theory, Methods and Applications, 71, 1401-1407.

Aryal GR, Tsokos CP (2011). "Transmuted Weibull Distribution: A Generalization of the Weibull Probability Distribution." European Journal of Pure \& Applied Mathematics, 4, 89-102.

de Gusmão FRS, Ortega EMM, Cordeiro GM (2011). “The Generalized Inverse Weibull Distribution.” Statistical Papers, 52, 591-619.

Drapella A (1993). “The Complementary Weibull Distribution: Unknown or Just Forgotten?" Quality and Reliability Engineering International, 9, 383-385.

Johnson NL, Kotz S, Balakrishnan N (1994). Continuous Univariate Distributions. Wiley, New York.

Keller AZ, Goblin MT, Farnworth NR (1985). "Reliability Analysis of Commercial Vehicle Engines." Reliability Engineering, 10, 15-25.

Khan MS, King R (2013). "Transmuted Modified Weibull Distribution: A Generalization of the Modified Weibull Probability Distribution." European Journal of Pure and Applied Mathematics, 6, 66-88.

Mudholkar GS, Kollia GD (1994). "Generalized Weibull Family: A Structural Analysis." Communications in Statistics - Theory and Methods, 23, 1149-1171.

Murthy DNP, Xie M, Jiang R (2004). Weibull Models. John Wiley \& Sons.

Shaw WT, Buckley IR (2009). "The Alchemy of Probability Distributions: Beyond Gram-Charlier Expansions, and a Skew-Kurtotic-Normal Distribution from a Rank Transmutation Map." arXiv preprint, p. arXiv:0901.0434.

Swain JJ, Venkatraman S, Wilson JR (1988). "Least-Squares Estimation of Distribution Functions in Johnson's Translation System.” Journal of Statistical Computation and Simulation, 29, 271-297. 


\title{
Affiliation:
}

Faton Merovci

Department of Mathematics,

University of Prishtina

Prishtinë, 10000

Kosovo

E-mail: fmerovci@yahoo.com

Ibrahim Elbatal

Institute of Statistical Studies and Research

Department of Mathematical Statistics

Cairo University

Egypt

E-mail: i_elbatal@staff.cu.edu.eg

\author{
Alaa Ahmed \\ Institute of Statistical Studies and Research \\ Department of Mathematical Statistics \\ Cairo University \\ Egypt \\ E-mail: Alaa_mnn@yahoo.com
}

Austrian Journal of Statistics

published by the Austrian Society of Statistics

Volume 43/2

June 2014 http://www.ajs.or.at/

http://www.osg.or.at/

Submitted: 2013-10-08

Accepted: 2014-03-01 